Running head: Analysis of Wave propagation

\title{
Characterization of wave propagation in thin laminated plates
}

\section{Chao $\mathrm{Du}^{1}$, Qing-Qing $\mathrm{Ni}^{*}{ }^{2}$ and Toshiaki Natsuki ${ }^{3}$}

${ }^{1}$ Interdisciplinary Graduate School of Science and Technology, Shinshu University, Ueda City, Nagano, Japan

$*^{2(a)}$ Key Laboratory of Ministry of Education, Zhejiang Sci-Tech University, Xiasha Higher Education Zone, Hangzhou 310018, China

(b) Dept. of Functional Machinery and Mechanics, Shinshu University, 3-15-1 Tokida, Ueda 386-8576, Japan

${ }^{3}$ Dept. of Functional Machinery and Mechanics, Shinshu University, 3-15-1 Tokida, Ueda 386-8576, Japan

* Corresponding author

E-mail: niqq@shinshu-u.ac.jp

Fax: +81-268-215438

Tel: +81-268-215438

\begin{abstract}
The formulae including the in-plane stiffness and bending stiffness coefficients, and coupling effect in out-plane were presented for analyzing characterization of wave propagation. Using the present formulae, the wave velocities can be calculated in any case of laminated plates including non-symmetric lamination. In this study, the characteristics of wave propagation in thin laminated plates were investigated in detail. Five modes including symmetric modes and anti-symmetric modes were obtained from the proposed formulae. For each mode, the wave velocities of laminated plates were investigated in different conditions such as plate thickness, stacking sequence and vibrational frequency.
\end{abstract}

\section{Keywords}

Wave propagation; Laminated plates; Lamb wave; Symmetric mode; Anti-symmetric mode 


\section{Introduction}

In recent years, thin plate structures have been widely applied in many fields including aircraft and spacecraft external skins and pressure vessels due to weight and energy-saving. However, some damages like matrix cracking, delamination, transverse cracking and fiber breakage are required to be detected in real-time. The energy released from these damages may give rise to small surface displacements and cause transient elastic waves in materials. Thus, the wave propagation is necessary to detect damage mechanism and to predict damage life in materials or structures. Wave propagation of thin plates usually takes the form of lamb waves [1], and can be divided into three types: One type is called symmetric mode because the deformations are symmetric about the mid-plane of the plates; the second type is called anti-symmetric mode due to the deformations with anti-symmetric about the mid-plane; the third type is called shear horizontal $(\mathrm{SH})$ mode, in which the transverse particle vibrations are horizontal to the plane. Lame et al. and Rayleigh et al. [2] have reported the propagation characteristics of the waves in a thin plate, which are dependent on the plate thickness and boundary conditions in addition to the elastic properties and density of the materials. For symmetric laminated plates, the formulae of wave velocity were presented by Tang et al. [3]. Yamada et al. have studied the source location of impact [4]. And also, by exciting waves and measuring the characterization of these waves propagating in materials, the mechanical properties of the materials could be determined $[5,6]$.

In this study, the valuation formulae of wave velocity for arbitrarily-laminated plates were derived based on first order shear deformation theory. Using the proposed formulae, the wave velocities can be calculated in any case of laminated plates including non-symmetric lamination. Moreover, the influences of the propagating direction, the plate thickness and the stacking sequence on the wave velocity were investigated in detail.

\section{Theoretical approach}

In this study, coupling stiffness and rotary inertia coefficients [7] are considered to develop the evaluation formula of the wave velocity in arbitrarily-laminated plates. Coordinate system of a laminated plate is shown in Fig. 1. The $x$ and $y$ axes are in the mid-plane, the $z$ axis is normal to the lamina with its origin at the mid-plane, $f$ indicates the direction of the fiber orientation, and $h$ is the thickness of the plate. $u, v$ and $w$ are displacement components along the $x, y$ and $z$ directions, respectively. According to the first order shear deformation theory of laminated plates, the displacements of the laminated plates is defined as

$$
\begin{aligned}
& u=u_{0}(x, y, t)+z \psi_{x}(x, y, t) \\
& v=v_{0}(x, y, t)+z \psi_{y}(x, y, t) \\
& w=w(x, y, t)
\end{aligned}
$$

where $u_{0}$ and $v_{0}$ are the mid-plane displacement components, and $\psi_{x}$ and $\psi_{y}$ are the rotation components along $x$ and $y$ directions, respectively. 
The constitutive relations related to the force and moment resultants can be given by

$$
\left[\begin{array}{c}
N_{x} \\
N_{y} \\
N_{x y} \\
M_{x} \\
M_{y} \\
M_{x y} \\
Q_{x} \\
Q_{y}
\end{array}\right]=\left[\begin{array}{cccccccc}
A_{11} & A_{12} & A_{16} & B_{11} & B_{12} & B_{16} & 0 & 0 \\
A_{12} & A_{22} & A_{26} & B_{12} & B_{22} & B_{26} & 0 & 0 \\
A_{16} & A_{26} & A_{66} & B_{16} & B_{26} & B_{66} & 0 & 0 \\
B_{11} & B_{12} & B_{16} & D_{11} & D_{12} & D_{16} & 0 & 0 \\
B_{12} & B_{22} & B_{26} & D_{12} & D_{22} & D_{26} & 0 & 0 \\
B_{16} & B_{26} & B_{66} & D_{16} & D_{26} & D_{66} & 0 & 0 \\
0 & 0 & 0 & 0 & 0 & 0 & A_{44} & A_{45} \\
0 & 0 & 0 & 0 & 0 & 0 & A_{45} & A_{55}
\end{array}\right]\left[\begin{array}{c}
\partial u_{0} / \partial x \\
\partial v_{0} / \partial y \\
\partial w / \partial y+\psi_{y} \\
\partial w / \partial x+\psi_{x} \\
\partial u_{0} / \partial y+\partial v_{0} / \partial x \\
\partial \psi_{x} / \partial x \\
\partial \psi_{y} / \partial y+\partial \psi_{y} / \partial x
\end{array}\right]
$$

where $Q_{x}$ and $Q_{y}$ are the force and moment resultants per unit length along $x$ and $y$ directions, respectively. The $A_{i j}$ are the in-plane stiffness coefficients, the $D_{i j}$ are the bending stiffness coefficients, and the $B_{i j}$ are the coupling stiffness in-plane considering the bending effects. $A_{i j}, B_{i j}$ and $D_{i j}$ are defined as

$$
\begin{aligned}
& \left(A_{i j}, B_{i j}, D_{i j}\right)=\int_{-h / 2}^{h / 2} Q_{i j}^{(l)}\left(1, z, z^{2}\right) d z \\
& A_{i j}=k_{i} k_{j} \int_{-h / 2}^{h / 2} Q_{i j}^{(l)} d z \quad i, j=4,5
\end{aligned}
$$

where the $Q_{i j}$ are the transverse shear stiffness [8]. The superscript $l$ refers to the layer number of the laminated plates. The $k_{i}$ and $k_{j}$ are shear correction factors with $k_{4}^{2}=k_{5}^{2}=k_{4} k_{5}=5 / 6$.

From the derivation of the classical governing equation for in-plane motion and the derivation of the equation, the equations of motion are given by

$$
\begin{aligned}
& \frac{\partial N_{x}}{\partial x}+\frac{\partial N_{x y}}{\partial y}=\rho^{*} \frac{\partial^{2} u_{0}}{\partial t^{2}}+R \frac{\partial^{2} \psi_{x}}{\partial t^{2}} \\
& \frac{\partial N_{x y}}{\partial x}+\frac{\partial N_{y}}{\partial y}=\rho^{*} \frac{\partial^{2} v_{0}}{\partial t^{2}}+R \frac{\partial^{2} \psi_{y}}{\partial t^{2}} \\
& \frac{\partial M_{x}}{\partial x}+\frac{\partial M_{x y}}{\partial y}-Q_{x}=R \frac{\partial^{2} u_{0}}{\partial t^{2}}+I \frac{\partial^{2} \psi_{x}}{\partial t^{2}} \\
& \frac{\partial M_{x y}}{\partial x}+\frac{\partial M_{y}}{\partial y}-Q_{y}=R \frac{\partial^{2} v_{0}}{\partial t^{2}}+I \frac{\partial^{2} \psi_{y}}{\partial t^{2}} \\
& \frac{\partial Q_{x}}{\partial x}+\frac{\partial Q_{y}}{\partial y}=\rho^{*} \frac{\partial^{2} w}{\partial t^{2}}
\end{aligned}
$$

and

$$
\left(\rho^{*}, R, I\right)=\int_{-h / 2}^{h / 2} \rho\left(1, z, z^{2}\right) d z
$$

where $\rho$ is the mass density.

Considering the directional dependence, the waves propagating in the plane of laminated plates have the forms 


$$
\begin{aligned}
& u_{0}=A \alpha_{x} e^{i[k(m x+n y)-\omega t]} \\
& v_{0}=A \alpha_{y} e^{i[k(m x+n y)-\omega t]} \\
& \psi_{x}=\Psi_{x} e^{i[k(m x+n y)-\omega t]} \\
& \psi_{y}=\Psi_{y} e^{i[k(m x+n y)-\omega t]} \\
& w=W e^{i[k(m x+n y)-\omega t]}
\end{aligned}
$$

where $k$ is the wave number. $m$ is the direction cosine between the wave propagation and the $x$ axis, and $n$ is the direction cosine between the wave propagation and the $y$ axis. $\omega$ is the circular frequency, and $A \alpha_{x}, A \alpha_{y}, \Psi_{x}, \Psi_{y}$ and $W$ are the amplitudes of the waves.

Substituting Eq. (6) into Eq. (4), the matrix of coefficients for symmetric and anti-symmetric modes is expressed as

$$
\left.\mid \begin{array}{cc:ccc}
M_{11} & M_{12} & M_{13} & M_{14} & 0 \\
M_{12} & M_{22} & M_{14} & M_{24} & 0 \\
\hdashline M_{13} & M_{14} & M_{33} & M_{34} & M_{35} \\
M_{14} & M_{24} & M_{34} & M_{44} & M_{45} \\
0 & 0 & M_{35} & -M_{45} & M_{55}
\end{array}\right]: \begin{aligned}
& 1 \\
& 0
\end{aligned}
$$

where 


$$
\begin{aligned}
& M_{11}=\left(A_{11} m^{2}+2 A_{16} m n+A_{66} n^{2}\right) k^{2}-\rho^{*} \omega^{2} \\
& M_{12}=\left[A_{16} m^{2}+\left(A_{12}+A_{66}\right) m n+A_{26} n^{2}\right] k^{2} \\
& M_{13}=\left(B_{11} m^{2}+2 B_{16} m n+B_{66} n^{2}\right) k^{2}-R \omega^{2} \\
& M_{14}=\left[B_{16} m^{2}+\left(B_{12}+B_{66}\right) m n+B_{26} n^{2}\right] k^{2} \\
& M_{15}=0 \\
& M_{21}=M_{12} \\
& M_{22}=\left(A_{66} m^{2}+2 A_{26} m n+A_{22} n^{2}\right) k^{2}-\rho^{*} \omega^{2} \\
& M_{23}=M_{14} \\
& M_{24}=\left(B_{66} m^{2}+2 B_{26} m n+B_{22} n^{2}\right) k^{2}-R \omega^{2} \\
& M_{25}=0 \\
& M_{31}=M_{13} \\
& M_{32}=M_{14} \\
& M_{33}=\left(D_{11} m^{2}+2 D_{16} m n+D_{66} n^{2}\right) k^{2}+A_{55}-I \omega^{2} \\
& M_{34}=\left[D_{16} m^{2}+\left(D_{12}+D_{66}\right) m n+D_{26} n^{2}\right] k^{2}+A_{45} \\
& M_{35}=i\left(A_{55} m+A_{45} n\right) k \\
& M_{41}=M_{14} \\
& M_{42}=M_{24} \\
& M_{43}=M_{34} \\
& M_{44}=\left(D_{66} m^{2}+2 D_{26} m n+D_{22} n^{2}\right) k^{2}+A_{44}-I \omega^{2} \\
& M_{45}=i\left(A_{45} m+A_{44} n\right) k \\
& M_{51}=0 \\
& M_{52}=0 \\
& M_{53}=-M_{35} \\
& M_{54}=-M_{45} \\
& M_{55}=\left(A_{55} m^{2}+2 A_{45} m n+A_{44} n^{2}\right) k^{2}-\rho^{*} \omega
\end{aligned}
$$

According to previous research $[3,9]$, it is known that $S$-matrix is regarded as the symmetric mode, while $A$-matrix is regarded as the anti-symmetric mode. In this study, these modes are involved in one matrix including other elements. Thus, the wave velocity can be calculated more exactly in any case of laminates, including non-symmetric laminates. The phase velocity $(\omega / k)$ can be obtained when the determinant is set equal to zero.

\section{Valuation of wave propagation}

In this study, the characterization of the wave propagation in symmetric laminated plates was valuated based on the proposed formula. The wave velocities were calculated with the properties shown in Table 1.

Figure 2 shows the velocity dispersion curves of a 16-ply unidirectional laminated plate in different direction of wave propagation. We assume that the fiber orientation is in the direction of $0^{\circ}$. It is well known that the velocity of symmetric mode is a constant, while the velocity of anti-symmetric mode has different values for changing frequency. $S_{0}$ and $S_{0}$ as shown in Fig.2 are symmetric modes, while $A_{0}, A_{1}$ and $A_{2}$ were anti-symmetric modes.

Comparing Fig. 2-a), 2-b) and 2-c), the influence of the propagating direction on the wave velocity is investigated. The velocities of $S_{0}$ mode are $1453 \mathrm{~m} / \mathrm{s}, 1887 \mathrm{~m} / \mathrm{s}$ and $1453 \mathrm{~m} / \mathrm{s}$ along the 
fiber direction, $45^{\circ}$ and $90^{\circ}$ directions, respectively. The velocities of $S_{0}$ mode are $4660 \mathrm{~m} / \mathrm{s}, 3555 \mathrm{~m} / \mathrm{s}$ and $2541 \mathrm{~m} / \mathrm{s}$, respectively. In these modes, the waves excite particle displacement components along not only propagating direction but also perpendicular to propagating direction. Generally, the wave of $S_{0}$ mode is called the quasi-in-plane shear wave, and the wave of $S_{0}$ mode called quasi-extensional wave. The quasi-extensional wave has larger component of its particle displacement in the direction of wave propagation [10]. The velocities of $A_{1}$ and $A_{2}$ modes occurred in the frequencies of over $453 \mathrm{kHz}$ and $457 \mathrm{kHz}$, respectively. It can be found that the velocities decreased sharply with increasing frequency. The $A_{0}$ mode has the smallest velocity in all of modes. Figure 3 shows the velocity curves of $A_{0}$ mode of a 16-ply unidirectional laminated plate in different propagating directions. At the same frequency, the wave velocity is the largest value when the wave propagates along the fiber orientation, and it has the smallest value in the direction perpendicular to the fiber orientation. However, the velocities of $A_{0}$ mode increase with increasing frequency and then reach a plateau.

Figure 4 shows the velocity dispersion curves of an 8-ply unidirectional laminated plate in the direction perpendicular to fiber. The velocities of $S_{0}$ and $S_{0}$ modes are $1453 \mathrm{~m} / \mathrm{s}$ and $2541 \mathrm{~m} / \mathrm{s}$, respectively, and are the same as that in a 16-ply unidirectional laminated plate. The velocities of $A_{l}$ and $A_{2}$ modes appear when the frequencies (called the critical frequency) are over $905 \mathrm{kHz}$ and 913 $\mathrm{kHz}$, respectively. Comparing with the results shown in Fig. 2-c), the critical frequencies in the 8-ply unidirectional laminated plate are about twice as large as those in the 16-ply unidirectional laminated plate. For $A_{0}$ mode, the velocity curves of the 8-ply plate with different thicknesses are shown in Fig. 5. It is clearly observed that the velocity of $A_{0}$ mode in a thick laminated plate is larger than that in the thin one when the frequency is low. However, all of the velocities increase with increasing frequency and hold the same value.

Figure 6 shows the velocity dispersion curves of a quasi-isotropic laminated plate $\left([45 /-45 / 0 / 90]_{2 \mathrm{~s}}\right)$ and a cross-ply laminated plate $\left([0 / 90]_{4 \mathrm{~s}}\right)$. The velocities of $S_{0}$ ' and $S_{0}$ mode are $1969 \mathrm{~m} / \mathrm{s}$ and $3510 \mathrm{~m} / \mathrm{s}$ for [45/-45/0/90 $]_{2 \mathrm{~s}}$, and $1453 \mathrm{~m} / \mathrm{s}$ and $3753 \mathrm{~m} / \mathrm{s}$ for [0/90] $]_{4 \mathrm{~s}}$, respectively. It is found that the stacking sequence affects the wave propagation of symmetric modes. For the frequency larger than $455 \mathrm{kHz}$, the velocities of $A_{1}$ and $A_{2}$ modes for [45/-45/0/90 $]_{2 \mathrm{~s}}$ and $[0 / 90]_{4 \mathrm{~s}}$ are close to the same value. The velocity dispersion curves of $A_{0}$ mode in plates with different stacking sequences are shown in Fig. 7. The velocities in the plates of $[45 /-45 / 0 / 90]_{2 \mathrm{~s}}$ and $[0 / 90]_{4 \mathrm{~s}}$ are just between $0^{\circ}$ and $90^{\circ}$ directions of unidirectional laminated plates.

\section{Conclusions}

According to the first order shear deformation theory, the formulae including the effects of the shear deformation and rotary inertia were derived to evaluate the velocity of wave propagation in the arbitrarily-laminated plates. Based on the present theoretical analysis, the influences of the plate thicknesses and the frequency on the velocities of wave propagation in the symmetric laminated plates were investigated. 
Five modes including symmetric modes $\left(S_{0}\right.$ and $\left.S_{0}\right)$ and anti-symmetric modes $\left(A_{0}, A_{1}\right.$ and $\left.A_{2}\right)$ were derived with the present formulae. The dispersion of symmetric modes is independent on the frequency of vibration, and the velocities for each mode were hardly affected by the thickness of laminated plates. For anti-symmetric modes, the velocities of $A_{0}$ mode increased with increasing frequency, while the velocities of $A_{1}$ and $A_{2}$ modes decreased. The frequencies, at which $A_{1}$ and $A_{2}$ modes occurred, were affected by the thickness of laminated plates. Furthermore, the results of the laminated plates with different stacking sequences were obtained and the velocity dependence of stacking angles was made clear.

The further study will be done to verify the theoretical results with experiments and to investigate the wide range of application to a delamination problem.

\section{Acknowledgements}

The work was supported partly by Grant-in-Aid for Global COE Program and the project for "Creation of Innovation Centers for Advanced Interdisciplinary Research Areas (Shinshu University)" in promoting science and technology from the Ministry of Education, Culture, Sports, Science and Technology of Japan.

\section{References}

1. Lamb, H. On Waves in an Elastic Plate. Proc. R. Soc. 1917, A93, 114-128.

2. Rayleigh, J. W. S. The Theory of Sound; Dover Publications: New York, America, 1945.

3. Tang, B., Henneke, E., Stiffler, R. Acousto-ultrasonics Theory and Application; Plenum Press: New York, America, 1988.

4. Yamada, H., Mizutani, Y., Nishino, H., Takemoto, M., Ono, K. Lamb Wave Source Location of Impact on Anisotropic Plates. European Conference on Acoustic Emission Testing 2000, 77-83.

5. Lu, Y., Ye, L., Su, Z., Yang, C. Quantitative Assessment of Through-Thickness Crack Size Based on Lamb Wave Scattering in Aluminum Plates. NDT \& E Int. 2008, 41(1), 59-68.

6. Kim, Y., Kim, D., Han, J., Kim, C. Damage Assessment in Layered Composites Using Spectral Analysis and Lamb Wave. Compos. 2007, B38, 800-809.

7. Whitney, J. M. Structural Analysis of Laminated Anisotropic Plates; Technomic Pu. Co.: Lancaster, Pennsylvania, 1980.

8. Goetschel, D. B., Radford, D. W. Analytical Development of Through-Thickness Properties of Composite Laminates. J. Adv. Mater. 1997, 37-46.

9. Jeong, H., Jang, Y. Wavelet Analysis of Plate Wave Propagation in Composite Laminates. Compos. Struct. 2000, 49, 443-450.

10. Prosser, W. H. The Propagation Characteristics of the Plate Modes of Acoustic Emission Waves in Thin Aluminum Plates and Thin Graphite/Epoxy Composite Plates and Tubes. NASA Technical Memorandum 104187, Baltimore, Maryland, 1991. 
Table 1 Material properties of GFRP

\begin{tabular}{ccccc}
\hline $\mathrm{E}_{1}(\mathrm{GPa})$ & $\mathrm{E}_{2}(\mathrm{GPa})$ & $\mathrm{G}_{33}(\mathrm{GPa})$ & $v_{12}$ & $\rho\left(\mathrm{g} / \mathrm{cm}^{3}\right)$ \\
\hline 38.05 & 10.45 & 4.03 & 0.32 & 1.91 \\
\hline
\end{tabular}




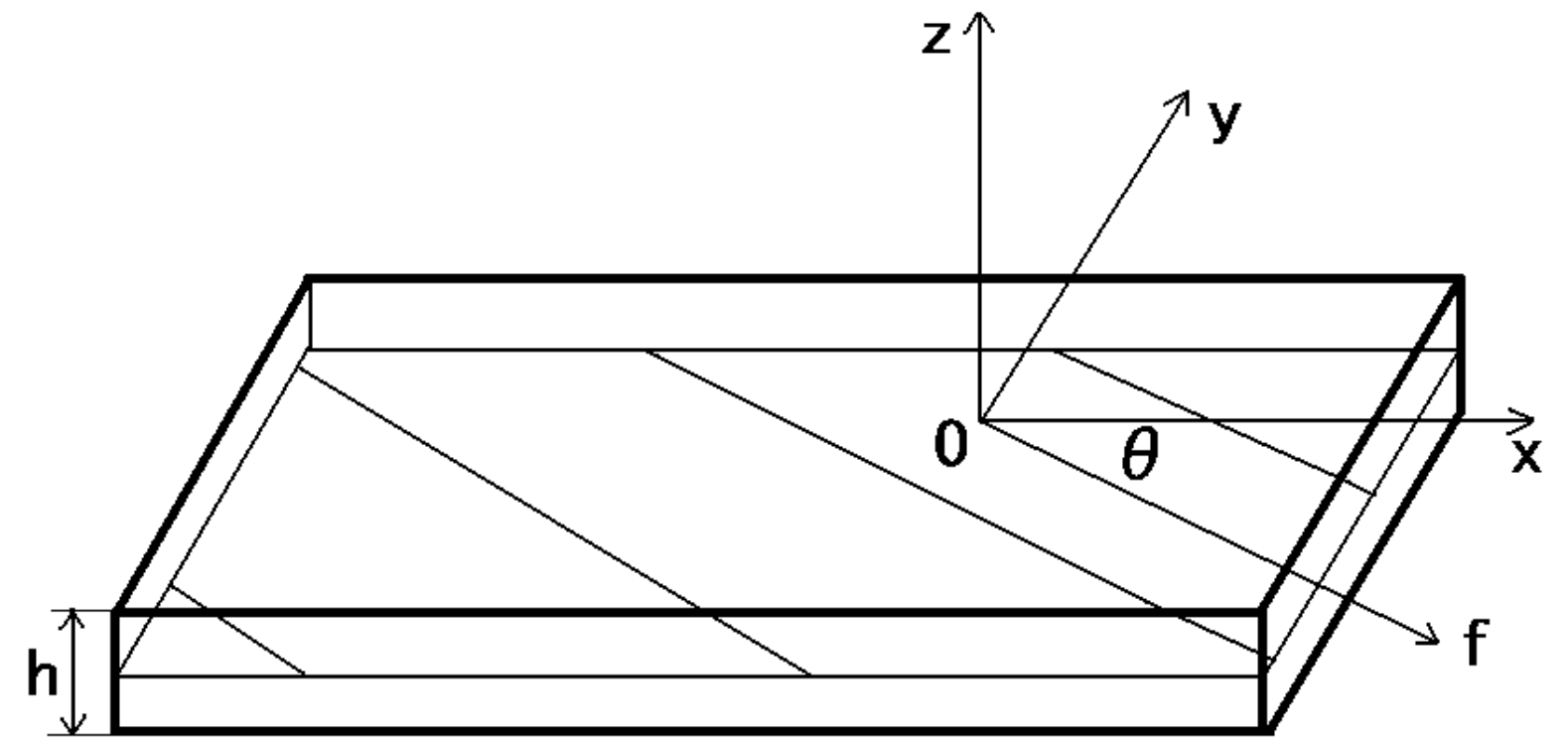

Figure 1 Coordinate axes of a laminated plate. 

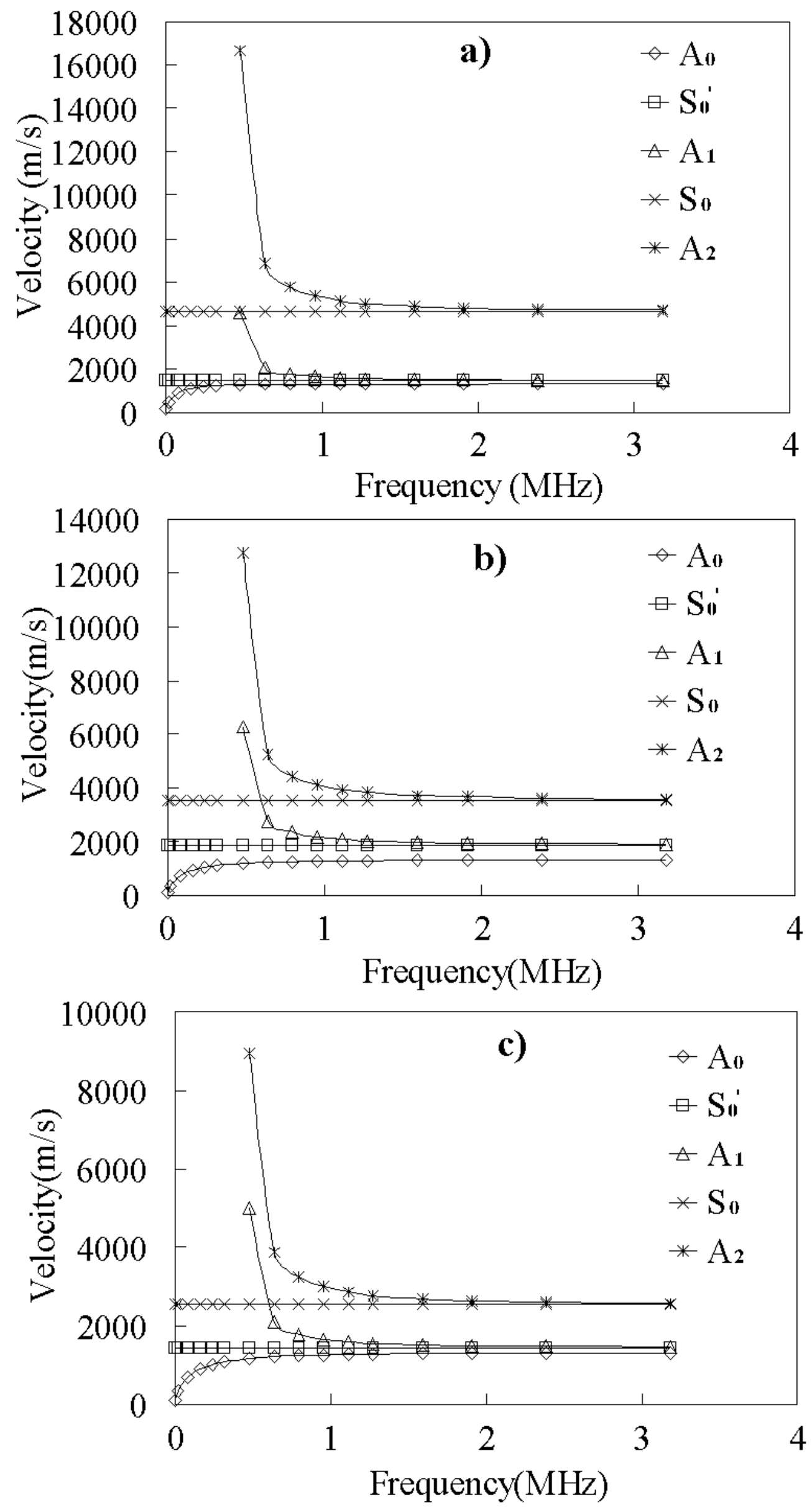
Figure 2 Dispersion curves of 16-ply unidirectional laminated plate.

(a) Wave propagating along fiber orientation. (b) Wave propagating along the direction of 45 degree with fiber orientation. (c) Wave propagating perpendicular to fiber orientation. 


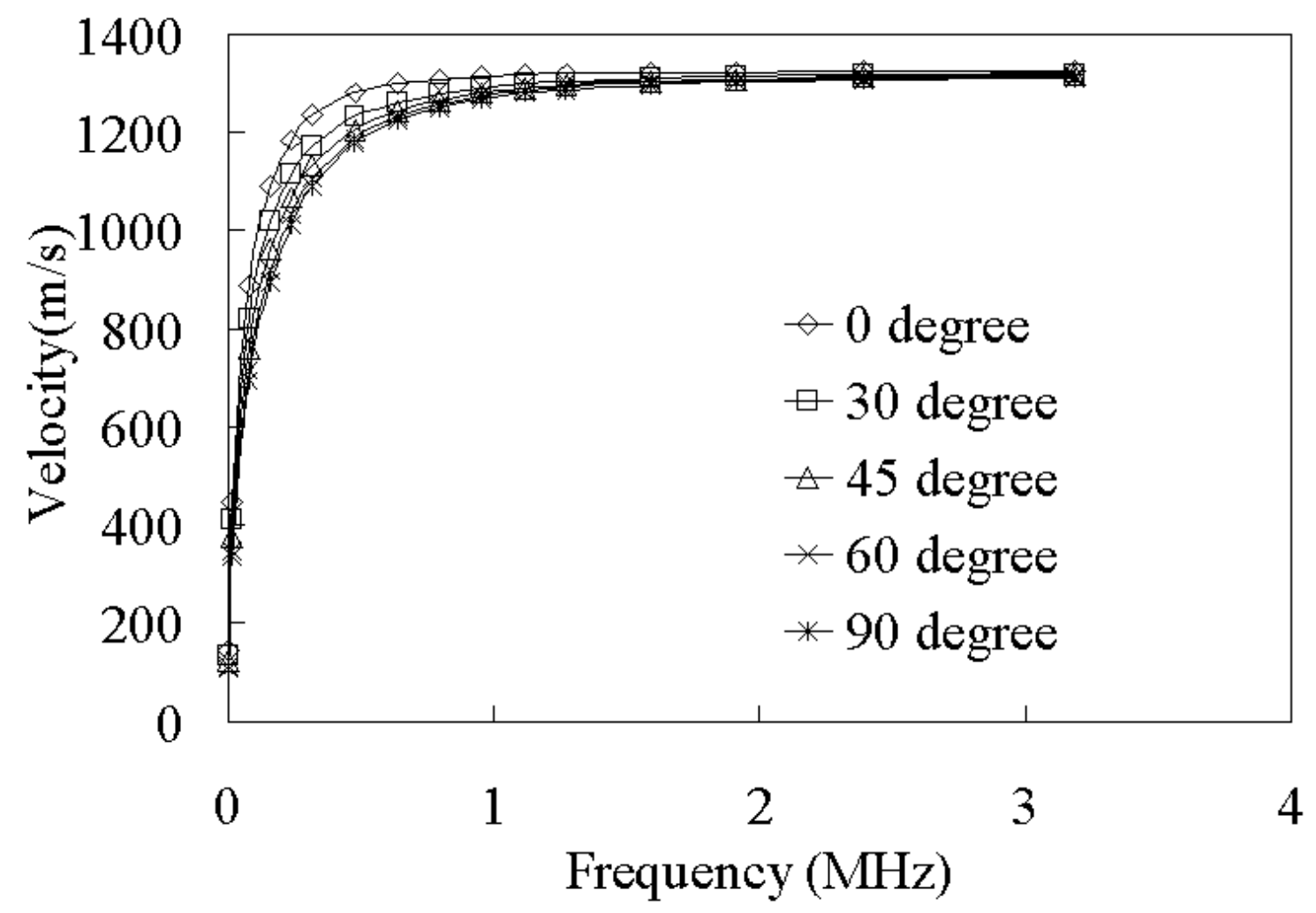

Figure 3 Velocity curves of $A_{0}$ mode of 16-ply unidirectional laminated plate in different propagating directions. 


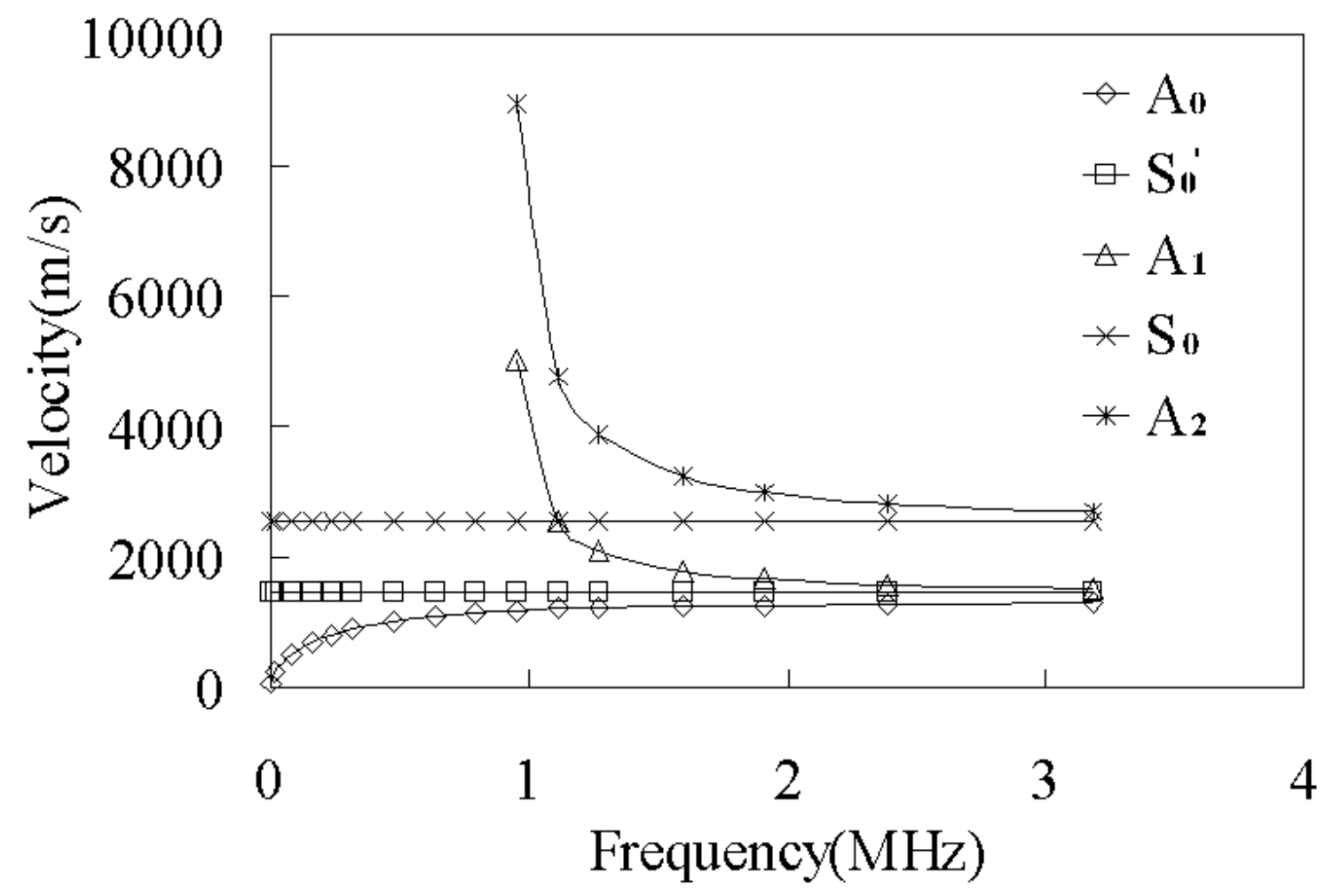

Figure 4 Dispersion curves of 8-ply unidirectional laminated plate in the direction perpendicular to fiber. 


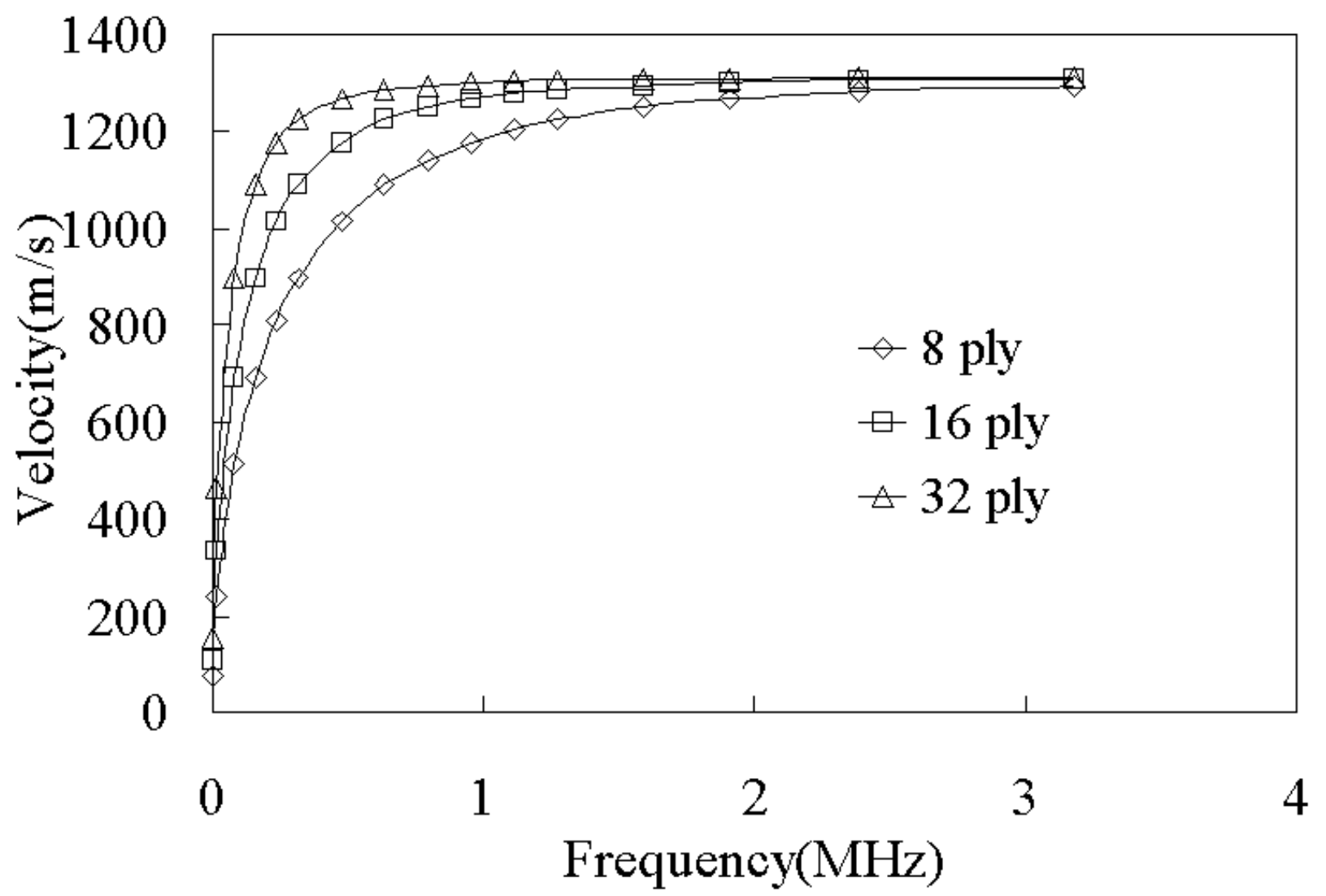

Figure 5 Velocity curves of $\mathrm{A}_{0}$ mode of unidirectional laminated plates in the direction perpendicular to fiber with different thicknesses. 

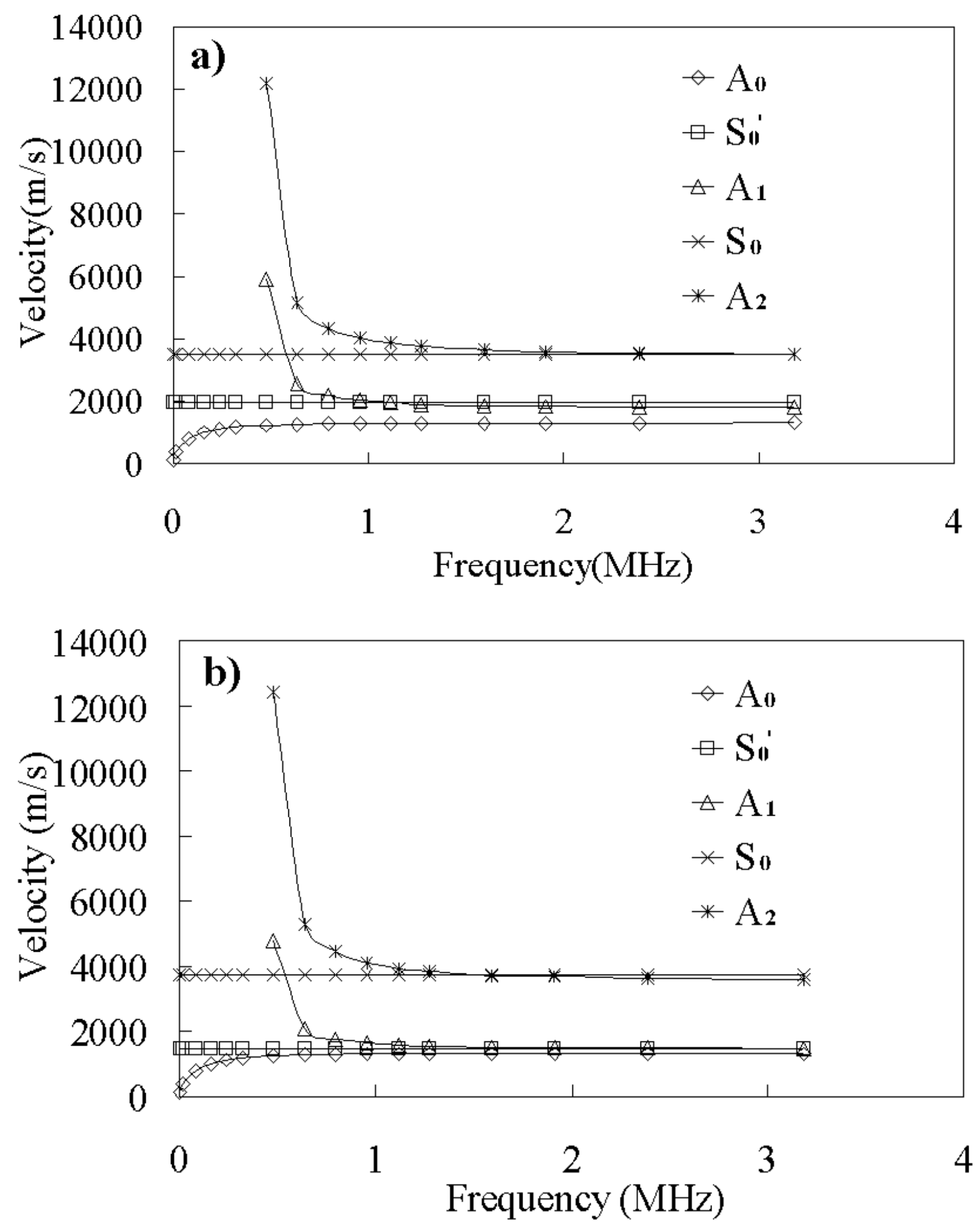

Figure 6 Influence of stacking sequence on dispersion curves along 0 degree direction of propagation.

(a) Dispersion curves of [45/-45/0/90 $]_{2 \mathrm{~s}}$ laminated plate. (b) Dispersion curves of $[0 / 90]_{4 \mathrm{~s}}$ laminated plate. 


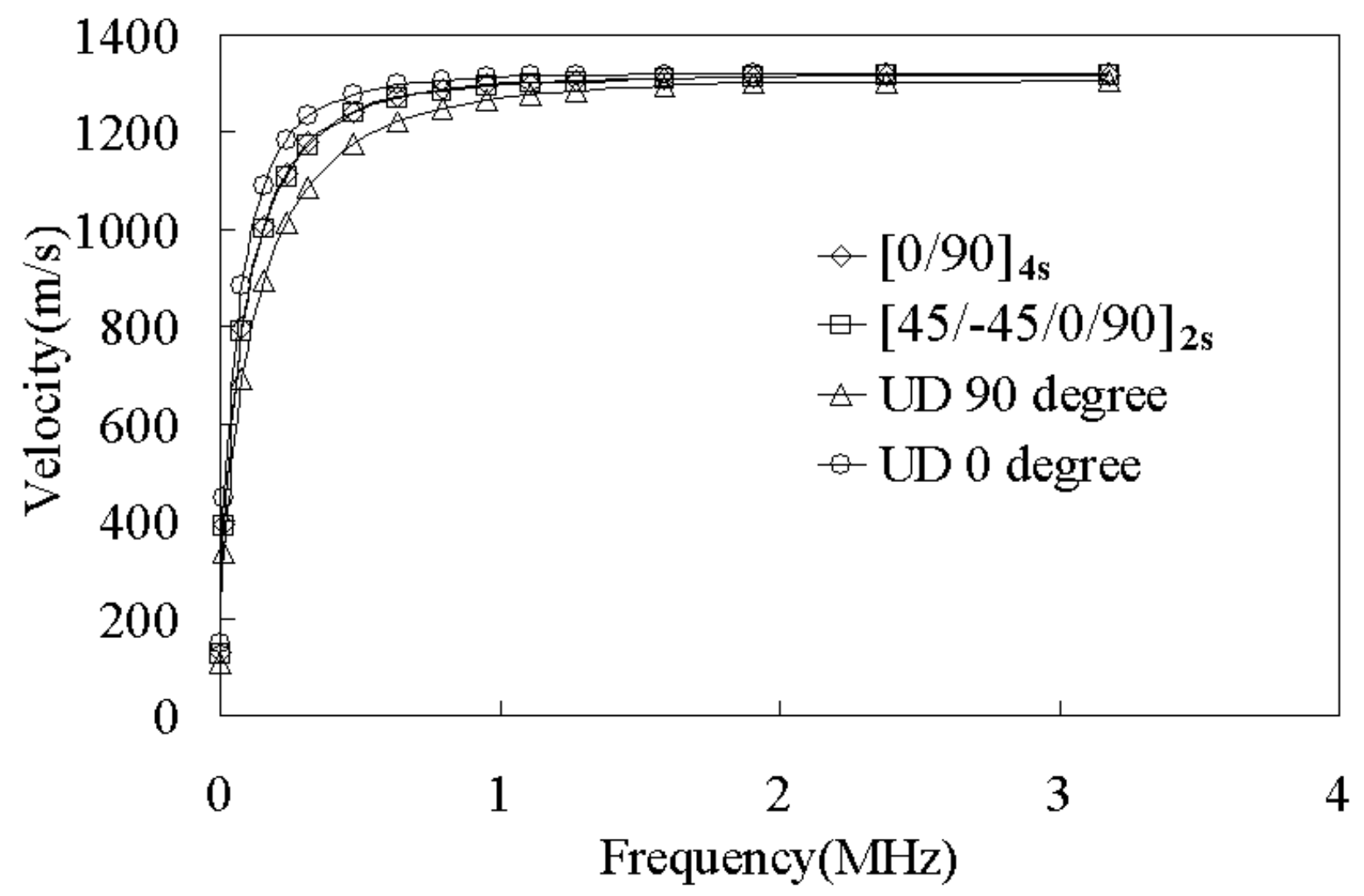

Figure 7 Velocity curves of $A_{0}$ mode of 16-ply laminated plate with different stacking sequences. 